\title{
Le classement en forêt de protection du massif de
}

\section{Haye}

Klassifizierung als Schutzwald des Hayemassivs

Jean-Pierre Husson

\section{OpenEdition}

Journals

Édition électronique

URL : http://journals.openedition.org/rge/1872

ISSN : 2108-6478

Éditeur

Association des géographes de l'Est

Édition imprimée

Date de publication : 1 janvier 2009

ISSN : 0035-3213

\section{Référence électronique}

Jean-Pierre Husson, "Le classement en forêt de protection du massif de Haye », Revue Géographique de l'Est [En ligne], vol. 49 / 2-3 | 2009, mis en ligne le 08 novembre 2010, consulté le 07 septembre 2020. URL : http://journals.openedition.org/rge/1872

Ce document a été généré automatiquement le 7 septembre 2020

Tous droits réservés 


\title{
Le classement en forêt de protection du massif de Haye
}

Klassifizierung als Schutzwald des Hayemassivs

\author{
Jean-Pierre Husson
}

"Mon territoire est autre chose que ces champs,
ces demeures et ces montagnes, mais ce qui les
domine et les noue"
Antoine de Saint- Exupéry.

Depuis toujours, l'agglomération de Nancy vit en lien avec le massif de Haye, tout à la fois territoire en connivence avec l'agglomération toute proche et également clôture de l'horizon à l'Ouest. Cette situation invite à se rapprocher de la citation de SaintExupéry, placée en avant-propos. Le massif est objet intrinsèque mais il est également trait d'union. C'est encore un lieu qui nous habite (Guérin-Pace, Filippova) dans la mesure où il est devenu une infrastructure de récréation et de ressourcement pour les citadins de son voisinage. Par ses lisières, sur lesquelles buttent aujourd'hui le tissu urbain et l'entrée de ville de Laxou, il forme un cadre amène et exposé au regard. Très longtemps, et les successions de cartes anciennes nous le confirment ${ }^{1}$, un espace naturel transitoire a existé entre la ville, les villages et la forêt. Au gré de l'histoire et des évolutions des densités, ce bandeau a évolué avec des vignes, vergers et jardins, des accrues forestières ou encore des friches puis aujourd'hui l'extension du bâti qui a plus ou moins investi les pentes en fonction de la nature des assises géologiques locales (calcaires, marnes, lignes de sources qui dominent des étages marneux mal consolidés, éventuellement des cavités karstiques et encore la prise en compte des impacts des minières aujourd'hui abandonnées). La décision du ministre J.-L. Borloo qui autorise à entamer une procédure de classement du massif en forêt de protection apparaît très novatrice, voire presque inédite car, jusqu'à maintenant, ce type de classement concerne avant tout des espaces menacés par l'érosion torrentielle (article L 411-1 du Code forestier et arrêté ministériel du 14 septembre $2009^{2}$. Cette décision va affecter un territoire patrimonial, chargé d'histoire, placé en continuité immédiate de l'agglomération, associé à tous les projets en cours, à la double échelle de 
l'agglomération et du périmètre du SCoT. Ces projets sont toujours conçus, situés et arrêtés entre la décision politique, la faisabilité technique et les souhaits exprimés par l'alchimie de la gouvernance faite avec les usagers, les associations (le principe de « soft law », de politique incitative et contractualisée pour faire l'adhésion, afin de faire aboutir un projet).

2 Le massif de Haye est un bel objet géographique dans la mesure où il forme un système, un lieu où se tissent de nombreuses actions et rétroactions entre des éléments mesurables comme la capitalisation de bois sur $\operatorname{pied}^{3}$ ou la valeur des locations de chasse. Il offre également de précieuses externalités, par exemple les bienfaits procurés par le filtrage de l'air, la percolation de l'eau, la protection des sols. Sa position invite encore à réfléchir au devenir de l'entrée de ville de Laxou - la Sapinière, à la mise en scène des lisières boisées et des bordures des linéaires routiers qui frôlent ou traversent ce massif. La gestion et la pérennité du massif passent par la mise en avant de l'intérêt général bien compris, anticipatif. Cette démarche donne une place importante à l'application des principes de précaution, à associer à toutes nos ambitions, pour tenter de faire des aménagements sinon durables, au moins transmissibles. Enfin, la mise en scène du massif (B. Boutefeu) est associée à la capacité de créer et canaliser l'événement autour de ce massif qui, au cours de son histoire a par exemple été le cadre de somptueuses scènes de chasse, en particulier sous Charles III. Cette étude cherche à apporter un supplément de sens à cette compréhension en cours en associant les territoires qui composent ce massif et les temps forts qui ont contribué à sa construction. Bref, il s'agit d'une contribution à verser au débat à organiser pour réussir ce classement qui doit être favorable à l'image et à la dynamique des territoires confédérés autour de cette vaste plaque forestière, en son cœur dominée par la forêt domaniale (Lanier), domaine privé de l'Etat et par définition inaliénable (ce qui ne fut pas toujours le cas, 189000 ha ayant été aliénés par le pouvoir entre 1830 et 1870).

Photo 1 (cliché André Humbert, CERPA). La marge entre forêt et ville, sur les anciennes terres de l'abbaye de Clairlieu, occupées par un lotissement massif depuis les années 1970.

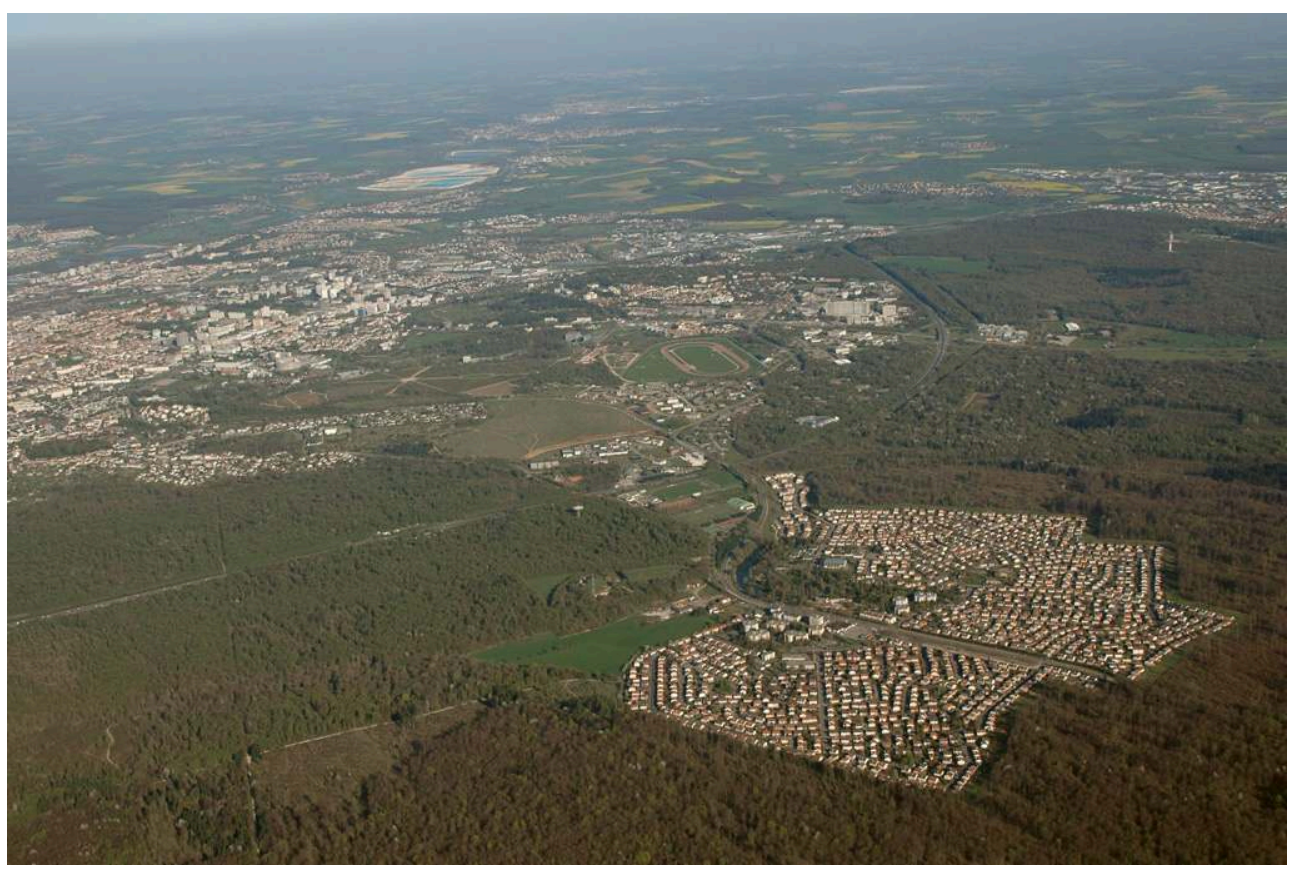




\section{I - Le massif de Haye, un bel objet géographique défini par la triple unité du lieu, du temps et de l'action}

Référer le massif à la métaphore théâtrale relève d'un emprunt à l'armature de la thèse de B. Boutefeu qui vient d'être reprise dans la parution d'un livre présenté dans ce numéro. Cette référence à la triple unité de lieu, temps et action invite bien à la mise en scène (ou du moins à la mise sous les projecteurs de l'actualité) du massif qui, si le classement en forêt de protection aboutit, sera engagé dans une aventure originale, presque inédite vu le peu de recul et d'expérience dont nous disposons sur ce sujet ${ }^{4}$; les forêts de protection étant historiquement celles qui sont associées à la mise en place de la politique de restauration de montagne (RTM) suite à la promulgation des lois de 1860 et 1882 .

4 Sur ses marges, le massif de Haye est occupé par des forêts communales (FC). Celles-ci ont été créées ou agrandies suite aux opérations de cantonnement, pour l'essentiel réalisées entre 1856 et 1940 (Husson, 1995) afin d'éteindre les droits d'usage qui grevaient alors les domaniales. Ces forêts, propriétés des collectivités, ont été dessinées en prolongement et en complémentarité des finages. Elles s'inscrivaient dans cette continuité fonctionnelle tant que les fonctions usagères, nourricières et affouagères furent importantes. Les processus de construction de ces forêts qui dessinent des transitions entre le cœur du massif et les marges désormais urbanisées ont été variés. Ils méritent d'être éclairés par quelques études de cas qui sont ici brièvement exposées. Par exemple, dès 1856, le cantonnement des bois de Chavigny fut obtenu. Au bois de Châtel situé au Sud-Est du village ont été ajoutées deux portions. Il s'agit de trois parcelles qui jouxtent la FC de Vandoeuvre sur Champelle et du bois des Clairs Chênes, aujourd'hui positionné en vis-à-vis du CHR, de l'autre côté de l'A 33. Cet exemple montre que les FC agrandies au cours du XIXe siècle sont surtout localisées sur les lisières, exposées au regard. A l'époque des Trente Glorieuses, elles ont pu être rognées pour loger des infrastructures. Ces bois sont parcellés, émiettés. Ce legs tend à compliquer leur gestion et invite aujourd'hui à mutualiser pour réaliser des aménagements cohérents. Ainsi, la commune de Chavigny qui dispose de 156 ha répartis en trois lots ne peut gérer isolément son bien et doit s'inscrire en solidarité avec ses voisins. On est bien loin de la situation initiale du traitement en taillis sous futaie (TSF) récolté à 24 ans, à l'exception du quart en réserve destiné à produire des billes de futaie (Bonnefont).

5 Toutes les forêts communales ont connu des processus de construction assez similaires à celui qui vient d'être évoqué, avec des bois situés en continuité du finage, possédés de temps immémorial par les communautés et agrandis par des achats, parfois avec des bois acquis par cantonnements. Cette genèse qui participe à l'extrême parcellisation des marges doit être prise en compte pour définir l'actuel périmètre de protection. D'autre part, le massif a connu des dynamiques spatiales qui vont dans les deux sens : aggradation des surfaces ou défrichements. Jusque vers 1960, l'agglomération n'atteint jamais l'orée de la forêt. Partout subsistent des espaces de transitions qui perdurent sous diverses formes. Il existe des espaces ouverts cultivés ou pâturés, par exemple autour des fermes Saint-Jacques et de Clairlieu. Il subsiste des zones enfrichées, là où les vignes ont périclité, parfois depuis plus d'un siècle, alors qu'au XVIIIe siècle, la vigne était l'ornement de la ville, du moins là où les coteaux sont bien exposés (avec de très nombreux toponymes cadastraux qui rappellent cette activité, par exemple Mi les 
Vignes à Laxou). Le reflux de la vigne (Husson 2004) s'est accompagné de la croissance des accrues qui ont fossilisé les terrasses où couraient naguère les cépages. Ces accrues obéissent également à la dynamique des bois et peuvent déborder les limites marquées par des bornages et des fossés. Cela concerne tous les terrains très pentus, avec des sols minces et une catena de pierres anguleuses. La carte des Naudin (Wagner) levée en deux temps, entre 1729 et 1732 (consultable sur le site de la Région Lorraine) ${ }^{5}$ montre bien que cette occupation viticole ancienne a constitué l'environnement familier de la ville pendant plusieurs siècles. In fine, ces remarques qui concernent à la fois la dynamique spatiale du massif et son découpage parcellaire désignent un espace qui n’a jamais été figé. Il faut tenir compte de cette donnée dans les réflexions à mener sur le zonage à réaliser car ces mouvements nous apportent un legs foncier délicat à gérer. Nombre de parcelles privées hérités de cette situation sont très petites, parfois en déshérence. Selon l'endroit où l'on se situe, le massif peut être sur la défensive alors qu'il était stable, voire conquérant avant que l'étalement urbain ne l'assaille. Ce legs foncier superpose des héritages de temps empilés. Il va poser la question de la reconnaissance des biens pour dessiner la limite du périmètre à protéger. L'arbitrage oscillera entre trois données. En premier, ce sera de tenter de créer des limites assez simples, même si elles ne sont pas dans la continuité des efforts de géométrification de nos prédécesseurs qui eurent à gérer de façon cartésienne les limites des massifs dont ils ne mesuraient pas toute la complexité. En second, il faudra que les limites définies puissent intégrer le principe de continuité écologique entre le dedans et le dehors, bref le maintien de corridors, d'écosystèmes complexes de marge, de transition à associer à la place de la nature dans l'agglomération. Enfin, il faudra localement tenir compte des réseaux visibles (possibilité d'élargir une infrastructure si la croissance des flux l'impose, si la visibilité du lieu est insuffisante ou si le risque local d'accidentologie est élevé) et des linéaires invisibles, enfouis (en particulier le positionnement des réseaux d'eaux propres et usées, de gaz, d'électricité s'il y a lieu). Cette question se pose localement avec les conduites d'eau et de gaz qui desservent la ZAE qui a réinvesti la partie ouest de l'ancienne base américaine après le retrait de la France de l'OTAN.

\section{II- L'unité pédologique et géologique du massif explique l'actuelle dominante de la hêtraie}

6 Le massif de Haye (Venet 1983) correspond pour l'essentiel au revers de la Côte de Moselle. D'ouest en est, on passe d'une altitude de $250 \mathrm{~m}$ sur la lisière ouest du massif pour atteindre $361 \mathrm{~m}$ à la ferme Saint-Jacques. Ce lieu localise l'ultime défrichement agricole réalisé sur le haut du revers, là où les sols sont les plus minces. Il occupe une position dominante surplombant Nancy qui s'étalait au pied de l'amphithéâtre avant d'escalader puis de repousser les lisières forestières quand celles-ci n'étaient pas fixées par l'inaliénabilité. La dalle du Bajocien, déclinée localement en étage inférieur, moyen et supérieur, est incisée de nombreux et courts ruisseaux anaclinaux qui naissent à partir d'une ligne de sources. Celle-ci correspond au toit du Toarcien, avec quasiment en continu la présence de l'Aalénien ferrugineux. Cette présence explique les nombreuses concessions qui, par le passé, furent autorisées sans toujours bien cohabiter avec la présence de la forêt, avec la mémoire des risques karstiques et enfin les captages d'eau utilisés par la ville avant que cette dernière ne puisse directement ponctionner la Moselle (Martin 2010). La dalle calcaire est sillonnée de nombreux 
vallons froids. Elle offre une mosaïque de stations en fonction de l'épaisseur des sols, en majorité des sols minces, rouges, décalcifiés, médiocres et qui reposent sur des calcaires parfois fracturés (Becker, Le Tacon, Timbal). Localement, la pente et l'exposition sont des paramètres importants pour expliquer l'originalité des vallons froids reconnaissables à des plantes bioindicatrices comme l'ail des ours. Les flux d'ouest dominants qui apportent avec eux humidité, nébulosité, précipitations créent des conditions favorables à la croissance du hêtre. Rappelons le témoignage d'Emile Moselly : "Le printemps revenait, hésitant, furtif, sans couleur et presque sans joie, grelottant sous des averses, entre des nuées grises » (Terres lorraines, 1907). Malgré la référence littéraire évoquée, le revers de côte est en fait assez peu arrosé, avec $728 \mathrm{~mm}$. $\mathrm{p} / \mathrm{an}$, une température moyenne annuelle de $9,5^{\circ}$ et une saison végétative hors gel qui dure 7 mois. L'indice Houzard P/T s'inscrit dans des valeurs presque toujours subhumides même si de sévères écarts peuvent subvenir, générant des stress hydriques qui s'inscrivent dans la croissance radiatique des arbres. La récurrence des étés secs, voire caniculaires inquiète. Cette donnée s'ajoute aux effets désastreux du passage de Lothar (1999)(Weisrock, Husson) sur une futaie alors très régulière, mature, exposée aux flux d'ouest. L'essentiel des parcelles à peuplement mature qui n'avaient pas été régénérées a été laminé par la tempête. Celle-ci réduisit à néant le travail d'une succession de forestiers qui s'étaient ici initiés au passage de la conversion dès Parade et Vicaire, à partir du Second Empire. Si depuis, les régénérations naturelles ont été bonnes, abondantes, il faut cependant prévoir de ne pas laisser se réinstaller une dynamique où le hêtre serait trop dominant sur les quelques 2000 hectares en reconstitution. Au contraire, il faut inventer, recréer une forte biodiversité dans le mélange des espèces pour anticiper sur les changements climatiques à attendre et donner plus de capacité de résistance et de rugosité au couvert, par rapport aux effets de tempêtes. 
Photo 2 (cliché Jean-Pierre Husson). Dominée par les vétérans survivants du passage de l'ouragan Lothar, une abondante régénération annonce le visage futur de la forêt de Haye.

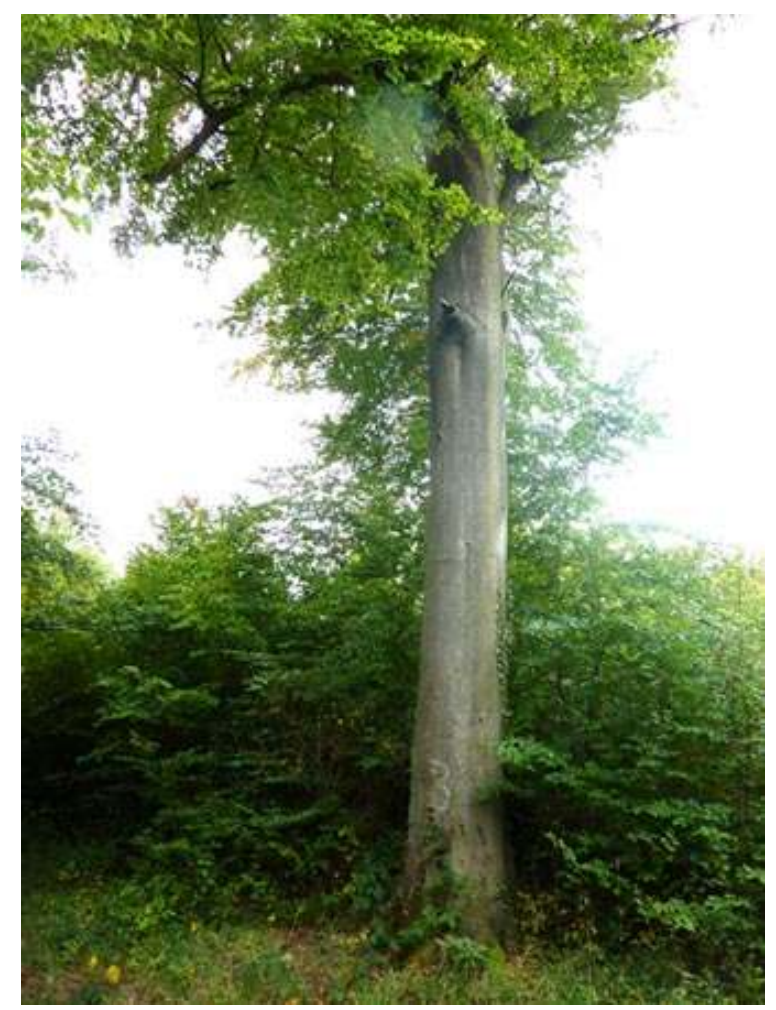

\section{III - Empilement et continuum historique}

L'actuel massif de Haye fonctionne comme une matrice qui nous livre désormais des traces qui y avaient été ensevelies ${ }^{6}$ puis conservées sous la forme d'un palimpseste redécouvert grâce au LIDAR. Sur cette matrice s'empilent des pages d'histoire, avec des territorialités qui conservent une certaine cohérence spatiale arrivée jusqu'à nous. Font exception les espaces rognés, essartés ou plus récemment défigurés par le tracé des linéaires logistiques surimposés. Ces derniers obéissent à la mise en place d'autres logiques que celles dictées par le sylvosystème en place et ses échelles spatiotemporelles spécifiques, celles des temps longs, ronds et prudents (Barrué-Pastor, Bertrand). Quatre étapes s'enchaînent, se télescopent, parfois cohabitent et plus souvent s'établissent dans les logiques d'exclusion de la précédente.

8 A l'origine, la forêt est nourricière, usagère et usufruitière, exploitée en collectif et dans l'intérêt prudent et partagé de communautés qui savent ne pas entamer le bienfonds. Ceci se traduit par la présence d'une forêt plutôt sur le retour, avec une dominante d'essences fruitières mais également des clairières et encore des marges parfois sur-ponctionnées, envahies d'espèces héliophiles, peu longélives qui répondent au toponyme de «fourasses». Excepté sur les marges, la ponction de bois demeure médiocre, le volume sur pied à tendance à être capitalisé. La vidange des bois relève de solutions de facilité, celles de débarder à partir des chemins sinueux implantés dans le fond des vallons froids, secs, parfois digités encadrés par des pentes raides qui dégagent des dénivelés allant jusqu'à $30 \mathrm{~m}$. Ces chemins sont identifiés par des toponymes médiévaux, par exemple La Crédence, Le Noirval. Ils forment la trame initiale (Blais, 
1934) sur laquelle se sont surimposées les autres trames viaires plus récentes, et qui les ont relégués au second plan.

Dès le XVIe siècle, le massif revêt une fonction cynégétique qui va s'imposer sur le reste. Cette activité explique la préservation du massif, ce qui se traduit par un précoce souci d'identification, de reconnaissance, de cartographie ${ }^{7}$. Le massif est aborné, fossoyé, découpé par des chemins rayonnants, rectilignes, tracés pour favoriser la pratique de la chasse à courre. Ces travaux routiers de grande ampleur concernent essentiellement les parties planes des plateaux; ce qui localement peut inviter à corriger des pentes. La chasse place la forêt à l'ombre du régalien. La construction de la Ville Neuve conforte cette position. En effet, en projetant de quadrupler la taille initiale de sa capitale et en s'affichant comme défenseur des valeurs de catholicité, Charles III qui accueille monastères et institutions charitables dans sa ville ${ }^{8}$ a également besoin de disposer d'énormes volumes de bois d'oeuvre (charpentes, pannes, etc.). La forêt de Haye, placée en proximité de la ville devient cette réserve (Guiot). Enfin, depuis 1559, la configuration géostratégique lorraine (Husson, 2003) fait que Nancy est située aux portes du royaume de France avec l'annexion des Trois Evêchés (dont Toul). Dans ce contexte, le massif devient également un objet de défense ; même si Nancy demeure pour cette période une place forte médiocre ou parfois démembrée (Choné, Fray, Thévenin).

10 La mise en place progressive des aménagements en taillis sous futaie se poursuit pendant tout le XVIIIe siècle. Cette méthode sylvicole provoque un changement paysager important, en imposant une construction en deux strates de la forêt. Malgré cela se dessine une certaine continuité avec la période précédente. Celle-ci se traduit par plusieurs constantes. Il s'agit d'abord de l'emprise croissante du régalien (en l'occurrence ici l'application de l'édit de Léopold en 1701, puis la Réformation associée à l'édit du Conseil de Finance de 1750) (Husson, 1991). Ensuite s'impose l'incessant travail de cartographie (Husson, 2008), d'inventaire, d'abornement et géométrification des lisières, le tracé de layons destinés à évacuer les bois et, ce qui diffère de la période précédente, le suivi des parcelles, le balivage des peuplements, bref, les actions nécessaires à la bonne conduite d'un peuplement en taillis sous futaie. In fine, cette pratique se traduit par une sélection à rebours au profit des essences les plus médiocres, le sol ayant été trop longtemps sollicité par des peuplements majoritairement conservés en phase juvénile?.

11 En 1824 est créée à Nancy l'Ecole forestière royale. De facto, les forêts d'Amance (1826) et de Haye (1834) vont être des terrains d'expérience pionnière et de proximité pour les élèves initiés par B. Lorentz aux balbutiements de la mise en conversion des bois (Blais, 1936). Ces bois sont donc au cœur de la grande querelle née du passage du taillis sous futaie à la futaie. Lorentz est en avance sur son temps, celui d'une conjoncture écotechnique médiocre, encore dominée par les principes et les valeurs du système protoindustriel gros consommateur de bois de feu. La conversion qui, au final, aboutit à l'enrichissement du capital sur pied, exigeait des sacrifices au propriétaire et amenait à réduire, encadrer ou faire disparaître les usages qui grevaient les bois au bénéfice des communautés et ayants-droit. La conversion du massif de Haye a connu une genèse chaotique. La décision de convertir (1834) a été très vite désavouée car le passage à la hêtraie réduisait les possibilités de récolter du bois de feu qui ne pouvait être trouvé ailleurs, faute d'avoir fini la percée du canal de la Marne au Rhin et d'avoir terminé la ligne ferroviaire desservant la ville. Il faut attendre 1859 pour que la conversion soit 
reprise, sur l'essentiel de la forêt domaniale. La conversion a permis de sortir de pratiques sylvicoles routinières et répétitives du balivage "en marquant là une coupe d'ensemencement, là une éclaircie, là une coupe préparatoire " (Blais, p. 56). Le legs transmis fut celui de la construction d'une futaie où la dominante du hêtre était très forte. Avant le passage de Lothar, une part importante de ce matériel sur pied, proche de la réalisation des coupes, formait une forte capitalisation de bois sous la forme de hêtraies presque équiennes. Les dégâts furent énormes dans ce type de parcelle.

Enfin, l'histoire de ce massif ne peut ignorer les effets de la partition de 1871. Nancy devient ville ouverte. Le système défensif mis en place par le général R. Séré de Rivières renforce le rôle des places fortes de Verdun, Epinal, Belfort et Toul (Husson, 2003). La défense de cette dernière s'appuie sur la côte, les buttes et avant-buttes, enfin sur le massif de Haye qui abrite des champs de tir, des espaces destinés aux entraînements. L'Armée défend la pérennité de la couverture forestière (Reitel). Enfin, en 1951, 520 ha de surface forestière sont concédés à l'OTAN pour établir une base américaine (RenardGrandmontagne 2010) . Après le départ de ces forces, l'espace fut restitué au ministère de l'Agriculture et partagé en trois secteurs : une ZA de 260 ha (dont 170 sont classés en zone UX), une zone de loisirs qui couvre 230 ha et enfin 20 hectares qui abritent l'école qui forme les techniciens forestiers (centre national de formation ONF de Velaine-enHaye).

Photo 3 (cliché André Humbert, CERPA). Enclavée dans le massif, l'ancienne base de l'OTAN (Nancy Ordnance Depot) reconvertie en zone périurbaine d'activités et de loisirs.

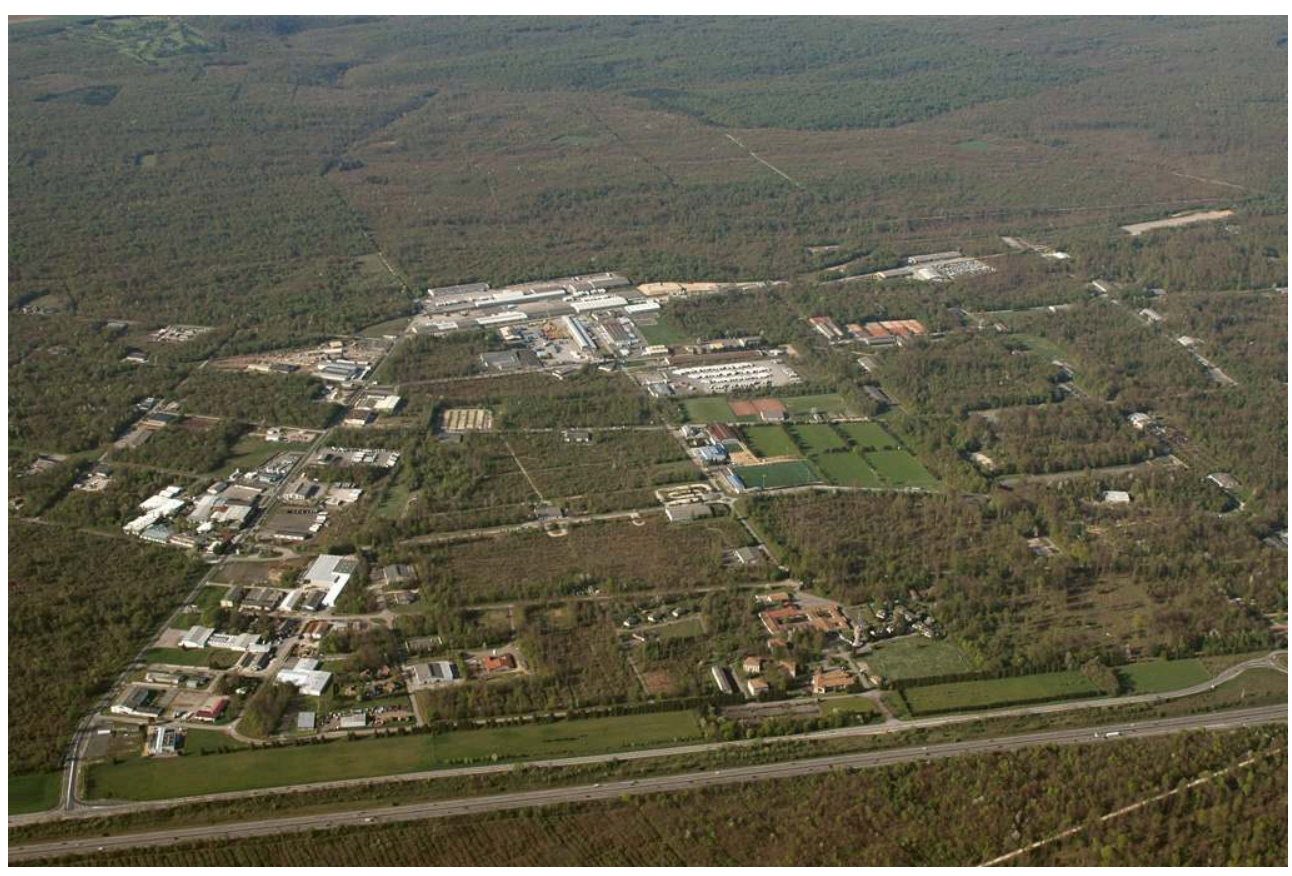

\section{IV - Un sylvosystème désormais hybridé par la ville}

L'hybridation relève certes de la métaphore mais signifie littéralement croisement et, par là, enrichissement à partir de deux souches. Entre l'agglomération et le massif de Haye fonctionne cet enrichissement, cette pratique d'apports respectifs l'un avec et par l'autre, ce qui se traduit par la volonté de dessiner un projet commun, initié par 
l'Association pour la Protection et la Sauvegarde du Massif de Haye. Ce choix est en rupture avec des épisodes passés où la forêt a, le plus souvent, été dominée, maltraitée par son voisin, avec en particulier un recul de son couvert forestier d'environ 1000 hectares par rapport à la période d'Avant Guerre. Le massif de Haye ne fait plus seulement partie de la mise en scène de l'agglomération. Il en est une réalité spatiale, une entité à part entière qui ne s'est cependant pas traduite par la finalisation d'une charte forestière de territoire (CFT) pourtant tentée en application de la loi d'orientation forestière traduite dans la circulaire du 15 février $2001^{10}$. Ce projet, non abouti, n'était pas terminé quand a été annoncée la décision d'autoriser le montage d'un dossier pour être classé en forêt de protection. Jusque là, des tensions, des blocages, voire la naissance d'un psychosystème (Arnould) risquaient de se nouer. En 2007 avait circulé une pétition intitulée « 10000 signatures pour sauver 10000 ha. ». Cette action était associée à l'élargissement du collectif de défense du massif. Le Grand Nancy se rallia alors au projet. Le 27 novembre 2007, le préfet impulse la volonté de changement, convoque dans les locaux de l'ONF du parc de Haye une réunion très ouverte et entame l'organisation de cinq groupes de travail pour préparer le dossier de demande d'inscription, pour cause d'utilité publique, du massif en forêt de protection ; ce qui interdit ultérieurement tout changement d'affectation.

Jusque là, le massif était partagé entre des enjeux cynégétiques, logistiques, récréatifs (assurés à titre gracieux pour les ayants-droit ou rétribués par les usagers), économiques et sylvicoles qui pouvaient être porteurs de conflit. Bref, tout cela fournit un ensemble de données qu'il fallait arbitrer et hiérarchiser. Par delà cette situation délicate à conduire, le passage de Lothar et son cortège d'immenses chablis et volis ont été un frein à la dynamique de changement qui était souhaitée. La récente impulsion apportée par la décision de janvier 2010 change la donne forestière. Cette dernière invite à élargir le jeu des acteurs impliqués. Surtout, elle encourage à travailler autrement, en croisant des échelles de réflexion et des approches différentes de ce qui se faisait avant. Elle tient davantage compte d'une ambition de multifonctionnalité qui évolue, se décline à géométrie variable selon plusieurs paramètres: les attentes exprimées localement, le cahier des charges et les investissements consentis, l'association du zonage réglementaire des PLU avec les prescriptions supracommunales édictées par le SCoT Sud $54^{11}$. Actuellement, les cinq ateliers initiaux sont passés au nombre de neuf. Ils ont pour but de piloter le chantier de préservation du massif de Haye. Il s'agit de réfléchir sur l'évolution des intercommunalités et leur rapport avec l'échelle du massif, la perception du dedans et du dehors ${ }^{12}$, les entrées de villes jouxtant le massif, les interfaces entre le bâti et les bois, les déplacements entre Nancy et Toul. A ces groupes de questions s'ajoute la poursuite de l'inventaire des richesses naturelles du massif et leur mise en réseau. Se pose encore la refonte des aménagements d'accueil (Moignieu), la révision de la charte du parc de loisir et la concession à l'Association sportive Nancy-Lorraine (A.S.N.L). Enfin, il faudra traiter du devenir de la ZAE qui a investi la partie ouest de la base de l'OTAN après 1966.

Cet aperçu montre qu'il va falloir aborder différemment, que par un passé proche, le massif. La proposition de classement invite à rebondir, en sachant que le zonage d'un territoire demeure une décision délicate à arbitrer, à la convergence de logiques variées. Les stratégies foncières pèsent sur l'avenir des marges laminées par le tracé à délimiter. Ce dernier devra être en cohérence avec les logiques écosystèmiques, entre autres celles des corridors. Les territoires aiment en effet se développer en ménageant 
des marges, des écotones, transitions, voire synapses. Il faudra encore réfléchir aux approches paysagères. Derrière tout cela se profilent aussi les devenirs des entrées de villes et leurs espaces commerciaux, la gestion quantitative et qualitative des flux en liaison avec les modes de transports retenus et les vitesses de circulation autorisées. Enfin, se pose la façon d'envisager la forêt. Quelle infrastructure de proximité représente-t-elle pour une agglomération qui s'inscrit encore dans une logique de sillon métropolisé transfrontalier ? Réussir ce projet invite à le hisser, l'éclairer par des événements, le célébrer, bref communiquer de façon ludique, mémorielle et culturelle à son sujet. La fête est un ressort essentiel de cette réussite (Di Méo, Husson 2010). Cette dernière passe sans doute par la valorisation de l'histoire du territoire concerné, ce qui donne du souffle, parfois de la magie et du respect pour les lieux. Cette démarche de communication doublée d'histoire porte les idées d'envie, de partage et de coquetterie, associées à un espace qui perd ainsi sa dimension banale (Bigando). Enfin, la situation du massif de Haye est hybride. Sa spécificité relève à la fois de sa taille et de son positionnement. Ce dernier en fait un espace vert de proximité alors que par sa surface, le massif obéit à la classique représentation d'une forêt à la fois vaste, productive et peu pénétrée par l'esprit urbain (Granet, Dobré).

Au contact de la ville, l'évolution et l'anticipation des devenirs possibles d'un massif conduit à énoncer plusieurs questions souvent intriquées et dépendantes les unes des autres. Il s'agit en particulier de la protection, de la lutte contre l'érosion probable de la biodiversité, des changements de comportements des usagers dans leurs pratiques récréatives, de la prise en compte des changements climatiques (Pourias). L'énoncé des spécificités du massif de Haye participe à la réflexion préalable sur le projet de classement en forêt de protection.

\section{V - Sur quelles attentes réfléchir?}

La préparation du dossier de classement se heurte à plusieurs difficultés. Le traitement de celles-ci mérite à la fois des arbitrages, de la concertation, de la diplomatie dans la façon de communiquer et de bâtir le projet (Boutefeu, Arnould). Ces questions peuvent se rassembler autour de trois principales questions: quels zonages opérer?, quels nouveaux projets établir?, quelle biodiversité promouvoir dans le contexte du changement climatique prévisible? La complexité des articulations entre ces objectifs invite à réfléchir en termes d'assemblages d'échelles spatio-temporelles découvertes puis approuvés par tous les acteurs qui ont à intervenir sur cet espace où se focalisent d'importants enjeux pour l'avenir.

18 Le classement en forêt de protection a pour préalable la définition d'un zonage sur lequel on ne reviendra pas. Ce linéaire devra être négocié, défini à la convergence de trois paramètres : en premier, la prise en compte de la valeur du foncier porté par la spéculation renforcée par le souhait de densifier l'espace bâti (voire le besoin de construire pour satisfaire aux critères de mixité urbaine non atteints), en second la cohérence écosystémique et la mise en scène paysagère. Dans le périmètre retenu, les contraintes sont drastiques. Cette situation invite donc à prévoir une approche anticipative du contenu que l'on souhaite donner à ce contact particulier tracé entre la ville et sa forêt. Ce tracé sera également délicat à mettre en place. Quand on prend la mesure de l'émiettement foncier sur les marges du massif, on recense quelques 2500 propriétaires, pour partie difficiles à identifier (propriétés en déshérence, micro 
propriétés). Ces propriétés subsistent sur le faisceau où sera tracé le zonage et peuvent donc être concernées par ce dernier.

Le tracé évoqué doit s'effectuer en menant une opération de co-écriture, de ré-écriture du devenir du massif, avec l'aval d'un nombre d'acteurs bien plus important que précédemment. Ceci signifie qu'il va falloir que les dits acteurs s'imprègnent de la culture des autres, que les notions de temps long et de prudence, qui sont essentielles dans la conduite d'un sylvosystème, soient respectées. Associer, voire tenter de coordonner le temps pressé et fiévreux de la ville avec la quiétude indispensable à la conduite d'un peuplement forestier invite probablement à beaucoup de modestie. Le passage d'une hêtraie dominante à une forêt pluri-strate, mélangée, pouvant davantage résister et s'adapter aux stress hydriques, amplifiés par le caractère filtrant de la dalle calcaire, est essentiel pour faire évoluer le contenu phytosociologique du massif.

Enfin, il faudra articuler les échelles de décision. Le massif n'est ni une île, ni une enclave mais un territoire qui vit avec les composantes qui l'entourent. Les étapes de l'histoire du massif, les tentatives de rédaction d'une charte forestière, enfin l'actuel montage du dossier de classement en forêt de protection dessinent un continuum, un fil d'Ariane pour nous aider à réussir une mutation attendue, souhaitée, faite dans le respect de l'intérêt général.

\section{BIBLIOGRAPHIE}

Arnould P. (2002) - Histoire et mémoire des aménagements forestiers. Ingénierie, Eau, Agriculture, Territoires. Numéro spécial Aménagement forestier. Antony, Cemagref éditions, p. 9-20.

Barrué-Pastor M., Bertrand G. (textes réunis) (2000) - Les temps de l'environnement. Toulouse, PUM , $544 \mathrm{p}$.

Becker M., Le Tacon F., Timbal J (1980) - Les plateaux calcaires de Lorraine. Types de stations et potentialités forestières .Nancy, ENGREF, $216 \mathrm{p}$.

Bigando E. (2004) - Entre le social et le sensible : l'émergence d'un paysage ordinaire. Paris, Bulletin de l'Association de géographes français, 2-2004, p. 205-218.

Blais R. (1934) - La question forestière en forêt de Haye. Nancy, Revue des Eaux et forêts, p. 621-625.

Blais R. (1936) - Une grande querelle forestière, la conversion. Paris, PUF, 88 p.

Bonnefont J.-C. (1996) - Chavigny au XVIIIe siècle : un village à l'apogée de la civilisation rurale en Lorraine. Nancy, Revue géographique de l'est, 1-1996, p.11-75.

Boutefeu B. (2009) - La forêt mise en scène. Attentes des publics et scénarios de gestion de la forêt. Paris, L'Harmattan, $290 \mathrm{p}$.

Boutefeu B., Arnould P. (2006) - Le métier de forestier entre rationalité et sensibilité. Nancy, Revue forestière française, 1-2006, p. 61-72.

Choné P., Fray J-L., Thévenin E. (1998) - Le Grand Nancy, histoire d'un espace urbain. Nancy, PUN, 207 p. 
Di Méo G. (textes réunis) (2001) - La géographie en fête .Gap, Géophrys, 265 p.

Granet A-M., Dobré M. (2009) - Les citadins et la forêt en France. Nancy, Revue forestière française, 5-2009, p. 521-534.

Guérin-Pace F., Filippova E. (textes réunis) (2008) - Ces lieux qui nous habitent. Identité des territoires, territoires des identités. La tour d'Aigues, éditions de l'Aube, 276 p.

Guiot N. (1959) - La forêt de Haye en Lorraine aux XVI et XVIIe siècles. Nancy, Annales de l'est, 4-1959, p. 283-312.

Houzard G. (1984) - Vers un classement des bioclimats des forêts caducifoliées françaises. Nancy, Revue forestière française, 5-2004, p. 362-374.

Husson J-P. (1991) - Les hommes et la forêt en Lorraine .Paris, Bonneton, 318 p.

Husson J-P. (1995) - Les forêts françaises. Nancy, PUN, 256 p.

Husson J-P. (2003) - Les empilements géostratégiques et leurs héritages : l'espace lorrain. Paris, Stratégique, 4-2003, p. 59-80.

Husson J-P. (2004) - La vigne dans l'agglomération de Nancy, de l'objet relique au projet de renaturation. Nancy, Revue géographique de l'est, 3-2004, p. 81-87.

Husson J-P. (2008) - La Réformation forestière en Lorraine Dans Lemonnier-Lesage V., Lormant F. (textes réunis) Droit, histoire et société. Mélanges en l'honneur de C. Dugas de la Boissonny. Nancy, PUN, 628 p., p. 429-440.

Husson J-P. (2010) - Le paysage révélé par l'évènement festif : pourquoi ?, comment ?, pour quels résultats? Neufchâtel, Congrès des Travaux historiques et scientifiques, mise en ligne en cours sur le site des CTHS.

Lanier L. (1998) - La forêt domaniale. Nancy, Mémoires de l'Académie de Stanislas, 1997-1998, tome XII, p. 351-364.

Martin E. (2010) - L'hydrosystème domestique et urbain à Nancy au XIXe siècle. Nancy, thès ede géographie historique, 422 p. + annexes.

Moigneu T. (2005) - Gérer les forêts périurbaines. Paris, Editions de l'ONF, 414 p.

Pourias J. (2009) - Un aperçu des problématiques d'actualité en foresterie urbaine : l'exemple des forêts urbaines nantaises. Nancy, Revue forestière française, 5-2009, p. 513-5520.

Reitel F. (1984) - Le rôle de l'Armée dans la conservation des forêts en France. Paris, Bulletin de l'Association de Géographes français. 502-1984, p. 143-154.

Renard-Grandmontagne C. (2010) - Les anciennes bases de l'OTAN en Lorraine : quelles réutilisations ? Paris, B.A.G.F, 3, p. 408-418.

Venet J. (1983) - Le massif de Haye. Nancy, ENGREF, 43 p.

Viard J. (2002) - Le sacre du temps libre: la société des 35 heures. La Tour d'Aigues, éditions de l'Aube, 219 p.

Wagner P-E. (direction)(2003) - Les Naudin entre Meuse et Vosges. Metz, catalogue de l'exposition réalisée à la médiathèque du Pontiffroy, 117 p. + un cahier couleur.

Weisrock A., Husson J-P. (textes réunis) (2000) - L'ouragan du 26 décembre 1999 dans l'Est de la France. Nancy, Revue géographique de l'est, 3-2000, p. 103-149. 


\section{NOTES}

1. La première carte du massif de Haye a été dressée en 1609 par Vannesson, à l'initiative du duc Charles III afin d'aménager ses parcours de chasse à courre.

2. Définition des espaces qui peuvent être concernés par un classement en forêt de protection (article L 411.1) « Les bois et forêts, quels que soient leurs propriétaires, situés à la périphérie des grandes agglomérations ainsi que dans les zones où leur maintien s'impose, soit pour des raisons écologiques, soit pour le bien-être de la population ».

3. Le massif de Haye demeure sur l'essentiel de sa superficie prioritairement producteur de bois. Il fournit de belles récoltes de hêtre mais également du chêne et des grumes de feuillus précieux (frêne, érables, merisiers). Il capitalise encore une importante réserve de petits bois en partie transformée en plaquettes destinées au chauffage des collectifs de la Ville haute.

4. Peu de massifs périurbains bénéficient de ce statut, par exemple les forêts de Rambouillet, Fontainebleau, Sénart.

5. http://www.chr-lorraine.fr/naudin/

6. Voir dans ce même numéro la contribution signée par M. Georges-Leroy, J. Bock, E. Dambrine, J-L. Dupouey.

7. Le massif de Haye a été maintes fois cartographié, assez rarement dans son intégralité, le plus souvent sur une partie de son territoire, afin de tracer une route, apporter une pièce justificative à un procès, redresser une lisière, etc. Parmi ces cartes : ADMM B 10980 (plan de 1613) et un grand nombre de belles cartes du XVIIIe siècle, dont : B 11904 (Les Baraques), B 11995 (Chaligny), B 11999 (Maréville).

8. Consulter le plan dressé par Claude de La Ruelle en 1611.

9. ADMM B 10694 Visite générale des bois du Roi dépendants de la maitrise de Nancy (1783). Ce procès-verbal montre une dominante de bois épuisés, trop ponctionnés, peu capables de capitaliser sur pied.

10. Extrait de la circulaire datée du 15 février 2001 : «La charte forestière de territoire est un outil d'aménagement et de développement durable des territoires ruraux, insérant davantage les forêts dans leur environnement économique, écologique, social et culturel ».

11. Le SCoT Sud 54 a été publié en septembre 2009. Son périmètre réunit 468 communes et concerne 550000 habitants. Il a pour objectif de fixer un «projet d'aménagement solidaire de dynamique pour les vingt prochaines années». Son rôle est renforcé par la promulgation du Grenelle 2. Le SCoT Sud 54 fait partie des 12 SCoT retenus à titre expérimental pour participer à la démarche SCoT-Grenelle.

12. Voir dans ce même numéro la contribution de Marc Galochet.

\section{RÉSUMÉS}

La conservation du massif de Haye est due à la succession de quatre fonctions : nourricière, cynégétique, stratégique qui s'ajoutent à la production de bois de feu. Aujourd'hui, l'inventaire et le diagnostic du massif sont indispensables pour réussir son classement en forêt de protection. Ce texte est une contribution à ce projet qui fait du massif une « infrastructure " périurbaine, un patrimoine à promotionner. 
Die Erhaltung des Hayemassivs ist durch die Auseinanderfolge von vier Faktoren bedingt: Ausser der Brennholzfunktion, die Nahrungs-, die Jagd- sowie die Strategiefunktion. Heute ist es notwendig auf Inventar und Diagnose zurückzugreifen, um die Klassifizierung als Schutzwald zu ermöglichen. Der vorliegende Text ist ein Beitrag zum Projekt, welches dazu führen soll das Hayemassiv als periurbane „Infrastruktur“ und als Natur- und Kulturerbe anerkennen zu lassen.

INDEX

Mots-clés : massif de Haye, forêt de protection, aménagement, patrimoine, biodiversité

Schlüsselwörter : Hayemassiv, Schutzwald, Raumordnung, Natur-und Kulturerbe, Biodiversität

\section{AUTEUR}

\section{JEAN-PIERRE HUSSON}

Département de Géographie, Université Nancy 2. 